\title{
Order Flow and the Bitcoin Spot Rate
}

\author{
K. H. McIntyre ${ }^{1} \&$ Kristine Harjes $^{2}$ \\ ${ }^{1}$ Department of Economics \& Business, McDaniel College, Westminster, MD, USA. \\ ${ }^{2}$ The Motley Fool, Alexandria, VA, USA. \\ Correspondence: K. H. McIntyre, Department of Economics \& Business, McDaniel College, 2 College Hill, \\ Westminster, MD 21157 USA. Email: mcintyre@ mcdaniel.edu
}

Received: April 5, 2016

Accepted: April 21, 2016

Available online: April 25, 2016

doi:10.11114/aef.v3i3.1574

URL: http://dx.doi.org/10.11114/aef.v3i3.1574

\begin{abstract}
Bitcoin is a decentralized, open-source cryptocurrency used to make private, peer-to-peer transactions anywhere across the world. Although the individuals involved are (mostly) anonymous, every Bitcoin transaction is a matter of public record; anyone can view every Bitcoin transaction ever made. Following the methodology developed by Evans and Lyons (2002), this paper adapts and estimates a FX microstructure model that emphases order flow, the difference between buyer- and seller-initiated trading volume, to the Bitcoin market Using a data set consisting of all major currency transactions occurring on the Mt. Gox exchange, our results are quite similar to prior microfinance research on traditional currencies insofar order flow is a significant determinant of Bitcoin spot rates.
\end{abstract}

Keywords: Bitcoin, order flow, asset pricing, FX microstructure, cryptocurrency

\section{Introducation and Overview}

As the number of Internet users grows on a daily basis, different industries including the financial services industry are being affected (Riasi, 2015). Bitcoin, an open-source digital currency used to make private, peer-to-peer transactions, can be considered as one of the most significant innovations in financial services industry in recent years, emerging as a direct result of the expansion of Internet accessibility across the globe. Designed by cryptographer Satoshi Nakamoto (2008) and launched in 2009, Bitcoin has simultaneously been called a disruptive asset that will fundamentally alter the international payments system and a financial oddity that will disappear once the novelty wears off. Regardless of which, if any, view is correct, Bitcoin has experienced rapid growth in visibility and interest from investors and the financial press, in addition to drawing the attention of financial regulatory agencies in the United States, Japan, and other countries. That said, Bitcoin has received limited quantitative attention beyond calculation of the usual financial market summary statistics - trading volume, market capitalization, transactions, and so on. This is not terribly surprising: the Bitcoin market is a small one-at the time of this writing, approximately U.S. $\$ 7$ billion in Bitcoin are in circulation — with limited links to macroeconomic fundamentals. ${ }^{1}$

This paper formally analyzes the early Bitcoin market by exploiting the fact that every Bitcoin transaction is recorded and publicly available. As such, Bitcoin is uniquely suited for analysis using the foreign exchange (FX) microstructure methods developed by Evans and Lyons (2002). These models emphasize the importance of order flow, the difference between buyer- and seller-initiated volume. In theory, order flow reflects information, and resulting behaviors, that influence currency valuations; that is, order flow is a measure of demand pressure. Empirically, this approach has been quite promising: order flow and exchange rates are strongly correlated, and quantitative microstructure models have done a remarkably good job tracking price fluctuations in a variety of traditional currencies (King et al., 2013). On a cursory level, a relationship between cumulative order flow and the U.S. dollar/Bitcoin exchange rate seems to mirror previous findings with standard currencies. As seen in Figure 1, order flow and the Bitcoin spot rate exhibit clear comovement and at +0.88 , share a near-perfect correlation. Microfinance theory on mainstream currencies would suggest that increases in buying pressure may be driving Bitcoin price increases.

We adeapt and estimate a FX microstructure model for the Bitcoin market, with promising results. Our results are roughly consistent with prior microstructure research on standard currencies insofar we find that order flow accounts for up to $40 \%$ of measured volatility in the Bitcoin spot exchange rate. In contrast, available fundamentals have minimal or no explanatory power for changes in bilateral Bitcoin prices. We view this as noteworthy for two reasons: first, our 
results suggest that from an empirical perspective, Bitcoin has more in common with standard currencies than perhaps meets the eye. Second, the success in explaining price fluctuations in a young, undeveloped, and very turbulent asset like Bitcoin points to the fundamental robustness of the of the order flow/microstructure approach to asset pricing.

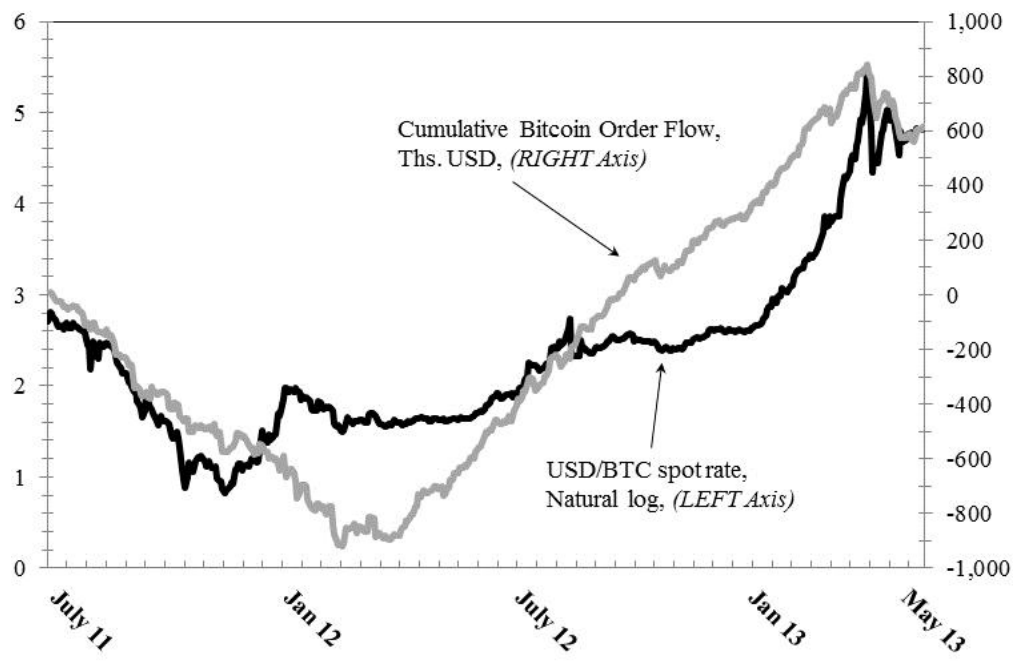

Figure 1. Bitcoin prices and order flow

The organization of the remainder of the paper is as follows: Section 2 briefly describes Bitcoin, the size and structure of the Bitcoin market, and discusses the relevance of using microfinance methods to analyze this market. Section 3 presents our data, empirical model, and results and Section 4 concludes.

\section{The Bitcoin Market}

\subsection{Basics}

Bitcoin is a pure fiduciary currency, operating with no sovereign central authority or banks. All transactions and Bitcoin creation are carried out and verified privately by the Bitcoin network. Entry into this network is free. As the identities of Bitcoin market participants are extremely difficult to ascertain, and because the Bitcoin network relies on sophisticated cryptographic methods to prevent counterfeiting and double-spending, Bitcoin is often referred to as a "cryptocurrency" in the popular and business press. Although its use as a currency in the traditional sense is to date limited, Bitcoin, like traditional currencies, is also used as a speculative financial asset (Velde, 2013).

One hallmark of the Bitcoin market is transparency: each Bitcoin transaction is recorded in the "blockchain," a database record of every transaction ever. The blockchain is in turn part of the Bitcoin "wallet," a computer file containing a series of encrypted keys on every Bitcoin user's computer; thus, any participant in the system can verify the validity of a transaction by tracing the history of a given Bitcoin or partial Bitcoin back its original creation. ${ }^{2}$

Any person involved in the Bitcoin market can conceivably see every transaction back to the initial creation of the currency, although the parties involved are anonymous. This peer-based monitoring inherent in the blockchain keeps the system in check and minimizes fraudulent activities such as double spending. Interestingly, that each and every computer with a Bitcoin wallet on it must also contain the blockchain means that any single, up-to-date user would be able to restore the entire system should it ever be destroyed. Additionally, the size of each transaction can be traced all the way back to the original creation of the bitcoin network. However, downloading the entire blockchain and updating it with each transaction is incredibly time consuming, and transactions can accordingly take up to a day to be processed and completed.

Formal management of the Bitcoin system is carried out through a public-key cryptographic system. That process works as follows: once a Bitcoin is officially purchased and the buyer has downloaded the wallet, the blockchain is updated by Bitcoin "miners," individuals who confirm and hash-encode a set of Bitcoin transactions into a "block" for addition to the blockchain. Miners are compensated through transactions fees and creating, or "mining" new Bitcoin in the compiled block. ${ }^{3}$ As such, Bitcoin miners serve two key purposes: they are de facto guarantors of Bitcoin security and are responsible for the creation of new Bitcoin. The majority of Bitcoin transactions, including transactions on the various Bitcoin exchanges, occur directly between end-users. Human intermediation in the Bitcoin market is extremely limited at present, or better, for the time period covered in this paper, and there are relatively few Bitcoin dealers or brokers.

Another hallmark of Bitcoin —indeed, a key attraction for many of its enthusiasts—is anonymity. Although each 
transaction is recorded in a publicly visible way and traceable back to an address, the identity behind a given address is extremely difficult to uncover; identity and address are anything but synonymous and a single person can have any number of addresses. This aspect of Bitcoin has been something of a blessing and a curse. On one hand, Bitcoin as an "online equivalent of a bag of cash" has benefits beyond basic privacy and civil liberties; it is a means, for example, for residents of unsafe and/or despotic nations to secrete wealth. On the other, the anonymity inherent in Bitcoin is attractive to those doing business in the darker corners of the Internet. ${ }^{4}$ Indeed, the potential to use Bitcoin for illegal activities like money laundering and drug dealing has tainted its reputation and is a commonly cited cause for concern about the future of the currency.

\subsection{Structure and Size}

While the Bitcoin market is miniscule compared to the broader foreign currency market, it has grown rapidly since Bitcoin's introduction in 2009, most notably in the 2013-2014 period. As noted in Figure 2, the total market value of all Bitcoin is at the time of this writing approximately U.S. $\$ 7$ billion. Daily Bitcoin volume is about U.S. \$50 million, compared to the U.S. $\$ 5$ trillion daily foreign currency market volume. Bitcoin is traded on peer-to-peer networks, with minimal intermediation and regulation (Plassaras, 2013). This is beginning to change, however, as more Bitcoin brokers and dealers are beginning to appear, and efforts are afoot in both the United States, Europe, and Japan to create a proper regulatory structure for Bitcoin. Like traditional currencies, most Bitcoin trading occurs in formal peer-to-peer exchanges. Through most of Bitcoin's early history, the Tokyo-based Mt. Gox exchange was the largest and best-known Bitcoin exchange, at its height handling approximately $70 \%$ of all Bitcoin trading until its spectacular collapse in early 2014. Of this, $80-90 \%$ all Bitcoin trading occurred into and out of the U.S. dollar. Other major currencies, namely the Euro, British pound, and Yen, in sum accounted for the lion's share of the remaining 10-20\% of all Bitcoin trading on Mt. Gox. ${ }^{5}$
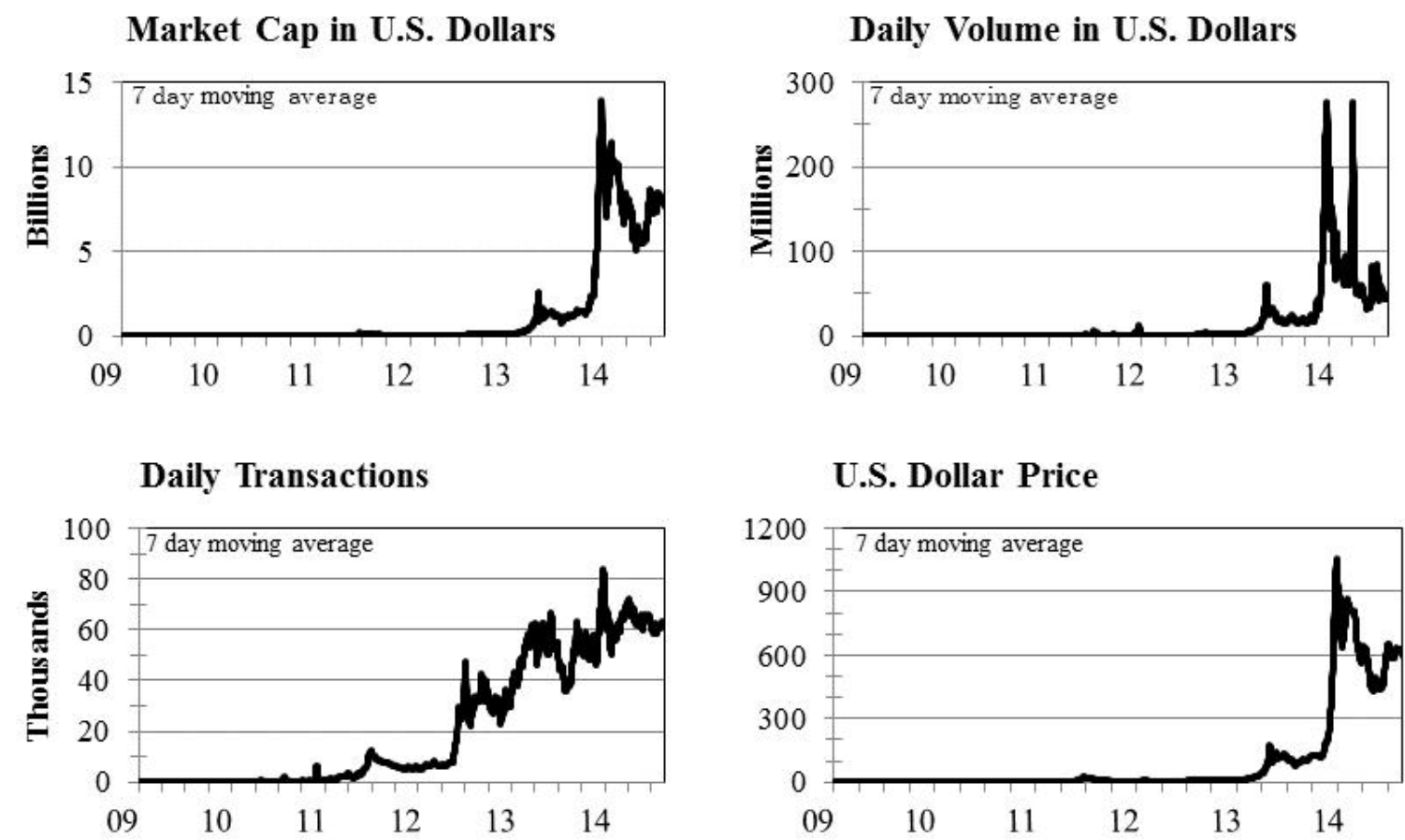

Figure 2. Bitcoin market snapshot, 2009-2014

A frequently noted feature of the Bitcoin market is volatility. Yermack (2013) reports that Bitcoin exchange rates are about twice as volatile as those of standard currencies, and that the Bitcoin exchange rate is largely uncorrelated with traditional exchange rates. A few examples may be illustrative here. When active daily Bitcoin trading began in April 2010, the U.S. dollar/Bitcoin exchange rate was approximately U.S. \$0.06 on the Mt. Gox exchange. At its height in late 2013, the U.S. dollar Bitcoin price had risen to over U.S. $\$ 1100$ (see Figure 2). In between, Mt. Gox experienced a serious hacking incident in 2011, multiple major technical glitches, and an investigation by the U.S. Department of Homeland Security, all of which suspended trading and roiled the Bitcoin market overall.

Mt. Gox collapsed spectacularly in February 2014 after the exchange reported it had lost nearly 850,000 Bitcoin, or about $7 \%$ of the entire Bitcoin supply, with a market value of over U.S. $\$ 470$ million as a result of a long-term hacking operation (Takemoto and Knight, 2014). It is noted that this hacking theft had gone undetected for years. Given that Mt. 
Gox accounted for such a large share of Bitcoin trading, the broader market response to its collapse bodes well for the long-term viability of the currency. The U.S. dollar Bitcoin price had fallen from its late-2013 high in advance of February 2014, and stood at about U.S. $\$ 700$ just prior to the Mt. Gox failure. The Mt. Gox insolvency pushed the Bitcoin price down to about U.S. $\$ 400$, which rebounded to U.S. $\$ 500$ by summer 2014 . The collapse of Mt. Gox has led to a consolidation of Bitcoin trading, and total Bitcoin trading volume is increasingly concentrated in larger exchanges. Moreover, brokers and dealers continue to enter the market. Accordingly, one can easily suggest that liquidity in the Bitcoin market is at an all-time high and is increasing.

\subsection{FX Microstructure Literature and Applications to Bitcoin}

The microfinance approach to exchange rates follows from the well-documented inability of traditional, fundamentals-based models of exchange rates to consistently track and forecast exchange rate movements over shorter-term time horizons. ${ }^{6}$ Instead of directly linking currency fluctuations to changes in broad macroeconomic fundamentals, the microstructure approach to exchange rates is predicated on the information, specifically the information regarding fundamentals and other factors relevant to currency values, available to individual market participants. This approach assumes that the information and beliefs relevant to currency valuations available at any given time is asymmetric among various agents (Evans, 2011). As such, the information relevant to currency values can be decomposed into public and private components, and the resulting behaviors stemming from innovations to each are important, individual drivers of exchange rate changes.

The key variable linking private information to currency valuations is order flow, the difference between seller- and buyer-initiated trading volume. As proposed in Evans and Lyons' (2002) portfolio balance model, order flow reveals and disseminates innovations in private information to others market participants, while changes in traditional, measured fundamentals such as interest rates, the money supply, output, and so on reflect new public information. Empirically, order flow has been shown to be strongly correlated with exchange rate fluctuations at higher frequencies over shorter-term time horizons (Evans and Lyons, 2002; Kitamura, 2010; Daníelsson et al., 2012) and additional work suggests macroeconomic news is indirectly reflected in order flow (Evans and Lyons, 2005 and 2008; Carlson and Lo, 2006; Evans, 2010; Chinn and Moore, 2011).

More recent work in microfinance has noted the distinctions among different types of order flow. Early microfinance research tended to focus on intradealer order flow, mainly due to a dearth of data on end-user or customer order flow. Although intradealer order flow contains information helpful to understanding currency movements, an increasing share of the microfinance literature stresses customer order flow (Reitz et al., 2007; Sager and Taylor, 2008; Lovcha and Perez-Laborda, 2010; Ding and Ma, 2013). There is good reason for this. Specifically, currency dealers typically open and close their day with a zero inventory position in foreign exchange. It follows that intradealer order flow should primarily reflect foreign exchange transactions initiated by end-users; dealer trading simply amplifies this process. Unfortunately, confidentiality concerns with banks and brokerages make the acquisition of customer order flow data difficult and as such, has limited this line of research.

The FX microstructure approach represents a natural starting point to study the Bitcoin market. Applying a traditional currency valuation model based on fundamentals to Bitcoin is a non-sequitur as there are few fundamentals associated with Bitcoin. There are no debt instruments, for example, denominated in Bitcoin, nor are there any real-valued fundamentals like output associated with the currency. The closest thing there is to a true fundamental is the supply of Bitcoin, which behaves more or less like a deterministic, logarithmic trend. That is, unlike typical money supplies, growth in the supply of Bitcoin is not influenced by policy or the state of the economy. It follows that private information and heterogeneous expectations should play an even greater role in determining Bitcoin prices.

Most importantly, all Bitcoin transactions, including those into and out of Bitcoin made on exchanges are publicly available. Included with information from exchanges is information on whether or not a given transaction was initiated as a bid or an ask order. That is, measuring order flow is a relatively straightforward process. Brokers and dealers, while present, are not the major players in the Bitcoin market that they are in regular currency markets, yet. As such, one can treat the Bitcoin order flow measured from a given exchange as the customer type.

That said, two points are in order. First and as previously mentioned, there are no traditional fundamentals save a mostly deterministic money supply associated with Bitcoin. This is noteworthy as microfinance models of exchange rates include a role for (bilateral) fundamentals, usually included as an interest rate spread due to data frequencies, in driving currency values. ${ }^{7}$ As previously noted, there are no debt instruments denominated in Bitcoin. Accordingly, it is problematic to formally incorporate bilateral fundamentals into any type of Bitcoin pricing model. Second, the pseudo-anonymous nature of the Bitcoin market makes distinguishing between agents virtually impossible. This may be a concern going forward given that order flows arising from different types of agents may have different impacts on a given exchange rate (Marsh and O'Rourke, 2005). We do not view these issues as particularly problematic for this study, however. Regarding the former issue, we can and do include unilateral fundamentals in the Bitcoin model we estimate; 
a sovereign interest rate, for example, represents an opportunity cost of holding Bitcoin as opposed to a traditional currency or asset, and may also reflect real-time expectations and public information regarding other sovereign fundamentals. Moreover, fundamental variables have been typically shown to add relatively little explanatory power in order flow models (Evans and Lyons, 2002; Cerrato et al. 2011). Regarding the latter, the typical Bitcoin exchange transaction averaged about U.S. $\$ 1,500$ over the latter half of 2013. The small transaction sizes suggests a lack of institutional investors of various sorts; anecdotal evidence found in the business and popular press suggests that for the time period studied in this paper, the Bitcoin market was primarily populated by individual investors. Therefore, it seems unnecessary to distinguish between various agents in the Bitcoin market at the present time.

\section{Order Flow and Bitcoin Price Movements}

\subsection{Model and Data}

Following the methodology detailed in Evans and Lyons (2002), we specify the following estimating equation:

$$
\Delta s_{t}=\psi_{X} \Delta X_{t}+\Psi_{F} F_{t}+\varepsilon_{t}
$$

In the above expression, $s$ denotes the natural logarithm of the standard currency/Bitcoin exchange rate; $\Delta s$ is the daily return to Bitcoin. $\Delta X$ denotes customer order flow with coefficient $\psi_{X}$, and $F$ is a vector of $k$ publicly-known fundamentals with coefficient vector $\Psi_{F}$ of length $k . \varepsilon$ is a mean-zero, i.i.d. error term.

Our data set consists of all U.S. dollar-, Euro-, British Pound-, and Yen-Bitcoin transactions occurring on the Mt. Gox exchange ending on May 23, 2013. The length of time series differs for each currency, with a start date corresponding to initial continuous daily trading in that currency. The U.S. dollar has the longest history, beginning on June 27, 2011. Our Euro time series begins on September 6, 2011, while the Pound and Yen start dates are December 28, 2011 and September 12, 2012, respectively. All Mt. Gox completed transactions were publicly available. Individual trade information includes the number of Bitcoin involved, the market value, and whether each trade was buyer- or seller-initiated. Bid and ask transactions were aggregated separately for each day. Daily order flow, $\Delta X$, is calculated as the difference between the value of bid trades and the value of ask trades, and is denoted in millions of U.S. dollars, Euros, and so on. Given the pseudo-anonymous nature of Bitcoin trading, we are unable to differentiate types of order flow. As noted previously, however, market characteristics and relatively small average transaction sizes make it very reasonable to treat all Bitcoin order flow as being of the consumer variety.

Descriptive statistics for Bitcoin order flows are found in Table 1. As expected, average and median order flows are close to zero. The standard deviation of Bitcoin order flows is largest for the U.S. dollar, which is expected given that the vast majority of Bitcoin trading occurs into and out of dollars. Order flow for the British Pound and Yen are both positively skewed and order flows for all currencies display a high degree of kurtosis. ${ }^{8}$ In the case of the British Pound and Yen, both currencies are thinly traded, and these results are the result of a handful of high-volume trading days. The last two columns of Table 1 report the first-order autocorrelation and corresponding p-value for each. With the exception of the Yen, daily order flows are positively autocorrelated. These autocorrelations are statistically significant, implying that past order flows carry some predictive power.

Table 1. Order Flow Descriptive Statistics

\begin{tabular}{lrrrrrrr}
\hline & & \multicolumn{5}{c}{ Standard } \\
& $\mathrm{N}$ & Mean & Median & Deviation & Skewness & Kurtosis & $\operatorname{ar}(1)$ \\
\hline U.S. Dollar & 499 & 0.001 & 0.002 & 0.0171 & -0.846 & 3.137 & 0.170 \\
Euro & 448 & 0.000 & 0.000 & 0.0010 & -0.250 & 3.369 & 0.264 \\
British Pound & 367 & 0.000 & 0.000 & 0.0001 & 2.178 & 14.589 & 0.180 \\
Yen & 177 & 0.000 & 0.000 & 0.0001 & 3.931 & 30.429 & -0.062 \\
\hline
\end{tabular}

Before continuing, a few additional words about the validity of these transactions data are in order. Specifically, we note that, if current commentary is true, the Mt. Gox exchange was victim to extensive theft operation during much of our sample period. We are not concerned about this as to date no suspicious activity or specific transactions have been identified that directly link to the alleged hacking theft. Of course there were such transactions, it is just likely that the thieves liquidated their Bitcoin positions in a way so as not to arouse suspicion. Accordingly, we argue that these criminal trades can be considered largely representative. Finally, we note that our sample ends in May 2013, well before Mt. Gox's February 2014 collapse.

Daily data on Bitcoin exchange rates at Mt. Gox was obtained online at www.bitcoincharts.com. Following Evans and Lyons (2002), various overnight nominal interest rates were used as fundamental drivers. Because there are no Bitcoin-denominated debt instruments, only interest rates for traditional sovereign currencies are employed as regressors, in lieu of international interest rate spreads. We test two interest rate specifications: the daily change in the 
interest rate, $\Delta i_{t}=i_{t}-i_{t-1}$, and the overnight yield with a one-day lag, $i_{t-1}$; both mirror Evans and Lyons (2002). For each sovereign currency we estimate (1) with different overnight interest rates. These yields are: the effective Federal Funds rate, the U.S. London Interbank Offered Rate (LIBOR), and the AA Financial Commercial Paper rate for the U.S.; the Euro LIBOR and the Euro Overnight Index Average (EONIA) rate for the Euro area; the British LIBOR and the Sterling Overnight Index Average (SONIA) for the U.K.; and the Yen LIBOR and the Uncollateralized Overnight Call Rate (OCR) for Japan.

\subsection{Results}

The results from estimating equation (1) are reported in Tables 2-5, with U.S. dollar results found in Table 2. Seven versions of (1) are estimated, incorporating different interest rate and trend combinations. For each specification, the order flow coefficient, $\psi_{X}$, is significant at at least the $1 \%$ level with the expected sign. That is, the positive sign on order flow signifies that net Bitcoin purchases leads to an increase in Bitcoin prices. The magnitude of the order flow coefficients, around 2.7 on average, suggests that on days with U.S. \$1 million more Bitcoin purchases than sales, the price of Bitcoin increases by about $2.7 \%$.

In contrast, overnight yields are rarely significant. Only the lagged Federal Funds rate has a non-zero coefficient. This sign, however, is correct; an increase in this yield decreases the U.S. dollar price of Bitcoin exactly as standard exchange rate theory predicts. Two final model variants introduced a trend term to proxy the relative supply of Bitcoin. (Over our sample period, the supply of Bitcoin relative to the U.S. M1 aggregate was more-or-less linear at monthly frequencies.) The trend term, however, is only significant once, when included with the lagged Federal Funds rate, but even then it is essentially meaningless with a magnitude at $7 \times 10^{-5}$.

The explanatory power of the U.S. dollar models is exclusively due to order flow, which explains about $41-43 \%$ of the variance in the Bitcoin returns. We note these results are consistent with those obtained when estimating order flow models to standard currencies (King et al., 2013). Even when significant, interest rates and the trend term contribute nothing to the model's $\mathrm{R}^{2}$ statistic. In fact, simply regressing the Bitcoin return on the various interest rate specifications always produces a zero $\mathrm{R}^{2}$. This finding, however, is not surprising. It is a well-known fact in international finance that macroeconomic fundamentals rarely carry little if any explanatory power for exchange rates at higher frequencies (Frankel and Rose, 1995). In addition, finding significant interest rates in microfinance models is often a hit-or-miss proposition and can be sensitive to model specification (Cerrato et al., 2011). And for the Bitcoin market, we again note that there are no fundamentals with any variance denominated in Bitcoin. Accordingly, we would not necessarily expect of see U.S. dollar-denominated fundamentals having a consistent, meaningful impact in this market.

Results for the Euro and British Pound are reported in Tables 3 and 4. These findings are for the most part analogous to the U.S. dollar results. The coefficients on order flow are strongly significant for the Euro and British Pound, and order flow is responsible for all of the models' explanatory power. Interest rates are never significant in any model specification that also includes order flow as a regressor. The main difference between the Euro and British Pound models and that of the U.S. dollar is less overall explanatory power. The Euro models, for example, produce $\mathrm{R}^{2}$ statistics that are less then one-half that of the U.S. dollar model; the British Pound models have an even lower R².

The Yen models perform relatively poorly. As noted in Table 5, the order flow coefficient is significant for the Yen, but at higher levels than for the U.S. Dollar, Euro, and British Pound. As usual, interest rates and a trend are not significant, and the 4-5\% overall explanatory power for these models is low. Although somewhat disappointing, these results are not surprising, given how thinly traded the Yen-Bitcoin market was and is. Daily ongoing Bitcoin-Yen trading commenced about a year after that for other currencies and the median number of daily Bitcoin trades into and out of Yen is 56 for our sample. Even today, Yen trading comprises a very small share of the Bitcoin market. It remains to be seen if a longer history and further market growth will improve the performance of this model variant.

Table 2. Regression Results, U.S. dollar

\begin{tabular}{|c|c|c|c|c|c|c|c|c|c|c|}
\hline & $\Delta X_{t}$ & $\Delta i_{t}^{F E D}$ & $\Delta i_{t}^{L I B O R}$ & $\Delta i_{t}^{C P O}$ & $\Delta i_{t}^{F E D}$ & $\Delta i_{t}^{F E D}$ & $\Delta i_{t}^{F E D}$ & TREND & $R^{2}$ & $D-W$ \\
\hline 1a & $\begin{array}{l}2.66 * * * \\
(0.21)\end{array}$ & $\begin{array}{l}-0.19 \\
(0.16)\end{array}$ & & & & & & & 0.41 & 1.80 \\
\hline $1 \mathrm{~b}$ & $\begin{array}{l}2.65^{* * * *} \\
(0.21)\end{array}$ & & $\begin{array}{l}-0.003 \\
(0.01)\end{array}$ & & & & & & 0.41 & 1.80 \\
\hline $1 \mathrm{c}$ & $\begin{array}{l}2.65^{* * *} \\
(0.21)\end{array}$ & & & $\begin{array}{l}-0.01 \\
(0.55)\end{array}$ & & & & & 0.41 & 1.80 \\
\hline 2 & $\begin{array}{l}2.65^{* * * *} \\
(0.21)\end{array}$ & & & & & & & & 0.41 & 1.80 \\
\hline $3 a$ & 2.73 *** & & & & $-0.15^{* *}$ & & & & 0.42 & 1.82 \\
\hline
\end{tabular}




\begin{tabular}{|c|c|c|c|c|c|c|c|c|c|c|}
\hline & $(0.22)$ & & & & $(0.07)$ & & & & & \\
\hline $3 b$ & $\begin{array}{l}2.65^{* * * *} \\
(0.22)\end{array}$ & & & & & $\begin{array}{l}0.0008 \\
(0.01)\end{array}$ & & & 0.41 & 1.80 \\
\hline $3 c$ & $\begin{array}{l}2.66^{* * * *} \\
(0.21)\end{array}$ & & & & & & $\begin{array}{l}-0.014 \\
(0.02)\end{array}$ & & 0.41 & 1.80 \\
\hline $4 a$ & & $\begin{array}{l}-0.15 \\
(0.23)\end{array}$ & & & & & & & 0.00 & 1.76 \\
\hline $4 b$ & & & $\begin{array}{l}-0.005 \\
(0.01)\end{array}$ & & & & & & 0.00 & 1.76 \\
\hline $4 c$ & & & & $\begin{array}{l}-0.88 \\
(0.71) \\
\end{array}$ & & & & & 0.00 & 1.77 \\
\hline $5 a$ & & & & & $\begin{array}{l}0.15^{*} \\
(0.09)\end{array}$ & & & & 0.00 & 1.77 \\
\hline $5 b$ & & & & & & $\begin{array}{l}0.02 * \\
(0.01)\end{array}$ & & & 0.00 & 1.77 \\
\hline $5 c$ & & & & & & & $\begin{array}{l}0.004 \\
(0.02) \\
\end{array}$ & & 0.00 & 1.76 \\
\hline $6 a$ & $\begin{array}{l}2.64 * * * \\
(0.22)\end{array}$ & $\begin{array}{l}-0.19 \\
(0.16)\end{array}$ & & & & & & $\begin{array}{l}8 \times 10^{-6} \\
\left(2 \times 10^{-5}\right)\end{array}$ & 0.42 & 1.80 \\
\hline $6 b$ & $\begin{array}{l}2.64^{* * * *} \\
(0.22)\end{array}$ & & $\begin{array}{l}-0.003 \\
(0.01)\end{array}$ & & & & & $\begin{array}{l}8 \times 10^{-6} \\
\left(2 \times 10^{-5}\right)\end{array}$ & 0.41 & 1.80 \\
\hline $6 c$ & $\begin{array}{l}2.64 * * * \\
(0.22)\end{array}$ & & & $\begin{array}{l}-0.004 \\
(0.57)\end{array}$ & & & & $\begin{array}{l}8 \times 10^{-6} \\
\left(2 \times 10^{-5}\right)\end{array}$ & 0.41 & 1.80 \\
\hline $7 \mathrm{a}$ & $\begin{array}{l}2.70 \text { **** } \\
(0.22)\end{array}$ & & & & $\begin{array}{l}-0.33 * * * \\
(0.09)\end{array}$ & & & $\begin{array}{l}7 \times 10^{-5 * *} \\
\left(2 \times 10^{-5}\right)\end{array}$ & 0.43 & 1.86 \\
\hline $7 b$ & $\begin{array}{l}2.64 * * * \\
(0.22)\end{array}$ & & & & & $\begin{array}{l}0.001 \\
(0.01)\end{array}$ & & $\begin{array}{l}8 \times 10^{-6} \\
\left(2 \times 10^{-5}\right)\end{array}$ & 0.41 & 1.80 \\
\hline $7 \mathrm{c}$ & $\begin{array}{l}2.65^{* * * *} \\
(0.22)\end{array}$ & & & & & & $\begin{array}{l}-0.01 \\
(0.02)\end{array}$ & $\begin{array}{l}8 \times 10^{-6} \\
\left(2 \times 10^{-5}\right)\end{array}$ & 0.41 & 1.80 \\
\hline
\end{tabular}

$N=499$; Date range: 27 June 2011 - 23 May 2013. Models are estimated using generalized method of moments correcting for autocorrelation up to order 4. The last column, marked $D-W$, reports Durbin-Watson statistics. Heteroscedasticity-consistent (Eicker-White) standard errors are in parentheses. $* * *=$ significant at $1 \%$; $* *=$ significant at $5 * ; *$ significant at $10 \%$.

Table 3. Regression Results, Euro

\begin{tabular}{|c|c|c|c|c|c|c|c|c|}
\hline & $\Delta X_{t}$ & $\Delta i_{t}^{L I B O R}$ & $\Delta i_{t}^{E O N I A}$ & $\Delta i_{+}^{L I B O R}$ & $\Delta i_{+}^{E O N I A}$ & TREND & $R^{2}$ & $D-W$ \\
\hline 1a & $\begin{array}{l}21.56 * * * \\
(3.85)\end{array}$ & $\begin{array}{l}0.05 \\
(0.03)\end{array}$ & & & & & 0.16 & 1.91 \\
\hline $1 b$ & $\begin{array}{l}21.56^{* * * *} \\
(3.85)\end{array}$ & & $\begin{array}{l}0.01 \\
(0.04)\end{array}$ & & & & 0.16 & 1.90 \\
\hline 2 & $\begin{array}{l}21.57 * * * \\
(3.85) \\
\end{array}$ & & & & & & 0.16 & 1.90 \\
\hline $3 a$ & $\begin{array}{l}21.21 * * * \\
(4.02)\end{array}$ & & & $\begin{array}{l}-0.01 \\
(0.01)\end{array}$ & & & 0.17 & 1.91 \\
\hline $3 b$ & $\begin{array}{l}21.23 * * * \\
(4.01)\end{array}$ & & & & $\begin{array}{l}-0.01 \\
(0.01)\end{array}$ & & 0.16 & 1.91 \\
\hline $4 a$ & & $\begin{array}{l}0.05 \\
(0.04)\end{array}$ & & & & & 0.00 & 1.74 \\
\hline $4 b$ & & & $\begin{array}{l}0.02 \\
(0.04) \\
\end{array}$ & & & & 0.00 & 1.73 \\
\hline $5 a$ & & & & $\begin{array}{l}-0.03 * \\
(0.02)\end{array}$ & & & 0.01 & 1.75 \\
\hline $5 b$ & & & & & $-0.02 *$ & & 0.01 & 1.75 \\
\hline
\end{tabular}


$(0.01)$

\begin{tabular}{|c|c|c|c|c|c|c|c|c|}
\hline $6 a$ & $\begin{array}{l}21.20 * * * \\
(5.29)\end{array}$ & $\begin{array}{l}0.05 \\
(0.03)\end{array}$ & & & & $\begin{array}{l}2 \times 10^{-5} \\
\left(3 \times 10^{-5}\right)\end{array}$ & 0.17 & 1.91 \\
\hline $6 b$ & $\begin{array}{l}21.19 * * * \\
(4.00)\end{array}$ & & $\begin{array}{l}0.01 \\
(0.04)\end{array}$ & & & $\begin{array}{l}2 \times 10^{-5} \\
\left(3 \times 10^{-5}\right)\end{array}$ & 0.16 & 1.91 \\
\hline $7 a$ & $\begin{array}{l}21.20 * * * \\
(4.00)\end{array}$ & & & $\begin{array}{l}-0.01 \\
(0.02)\end{array}$ & & $\begin{array}{l}3 \times 10^{-6} \\
\left(5 \times 10^{-5}\right)\end{array}$ & 0.17 & 1.91 \\
\hline $7 b$ & $\begin{array}{l}21.19 * * * \\
(4.01)\end{array}$ & & & & $\begin{array}{l}-0.01 \\
(0.02)\end{array}$ & $\begin{array}{l}1 \times 10^{-5} \\
\left(2 \times 10^{-5}\right)\end{array}$ & 0.16 & 1.91 \\
\hline
\end{tabular}

$N=448$; Date range: 6 September 2011 - 23 May 2013. Models are estimated using generalized method of moments correcting for autocorrelation up to order 4. The last column, marked $D$ - $W$, reports Durbin-Watson statistics. Heteroscedasticity-consistent (Eicker-White) standard errors are in parentheses. $* * *=$ significant at $1 \%$; $* *=$ significant at $5 * ; *=$ significant at $10 \%$.

Table 4. Regression Results, British Pound

\begin{tabular}{|c|c|c|c|c|c|c|c|c|}
\hline & $\Delta X_{t}$ & $\Delta i_{t}^{L I B O R}$ & $\Delta i_{t}^{S O N I A}$ & $\Delta i_{t}^{L I B O R}$ & $\Delta i_{t}^{S O N I A}$ & TREND & $R^{2}$ & $D-W$ \\
\hline \multirow[t]{2}{*}{$1 \mathrm{a}$} & $21.14 * * *$ & -0.43 & & & & & 0.07 & 1.55 \\
\hline & (5.12) & $(2.08)$ & & & & & & \\
\hline \multirow{2}{*}{$1 b$} & $21.33^{* * *}$ & & 0.04 & & & & 0.07 & 1.53 \\
\hline & (5.11) & & $(0.03)$ & & & & & \\
\hline \multirow[t]{2}{*}{2} & $21.16^{* * *}$ & & & & & & 0.07 & 1.54 \\
\hline & $(5.12)$ & & & & & & & \\
\hline \multirow{2}{*}{$3 a$} & $21.11 * * *$ & & & -0.08 & & & 0.07 & 1.55 \\
\hline & $(5.13)$ & & & $(0.10)$ & & & & \\
\hline \multirow{2}{*}{$3 b$} & $21.23^{* * *}$ & & & & -0.01 & & 0.07 & 1.54 \\
\hline & $(4.01)$ & & & & $(0.03)$ & & & \\
\hline \multirow[t]{2}{*}{$4 a$} & & -0.78 & & & & & 0.00 & 1.52 \\
\hline & & $(2.14)$ & & & & & & \\
\hline \multirow[t]{2}{*}{$4 b$} & & & 0.03 & & & & 0.00 & 1.51 \\
\hline & & & $(0.04)$ & & & & & \\
\hline \multirow[t]{2}{*}{$5 \mathrm{a}$} & & & & -.11 & & & 0.00 & 1.53 \\
\hline & & & & $(0.10)$ & & & & \\
\hline \multirow[t]{2}{*}{$5 b$} & & & & & -0.02 & & 0.00 & 1.52 \\
\hline & & & & & $(0.03)$ & & & \\
\hline \multirow{2}{*}{$6 a$} & $20.77 * * *$ & -0.96 & & & & $4 \times 10^{-5}$ & 0.07 & 1.56 \\
\hline & $(5.06)$ & (2.04) & & & & $\left(4 \times 10^{-5}\right)$ & & \\
\hline \multirow{2}{*}{$6 b$} & $21.00 * * *$ & & 0.04 & & & $4 \times 10^{-5}$ & 0.08 & 1.54 \\
\hline & $(5.06)$ & & $(0.03)$ & & & $\left(4 \times 10^{-5}\right)$ & & \\
\hline \multirow[t]{2}{*}{$7 \mathrm{a}$} & $21.44 * * *$ & & & 0.45 & & $1 \times 10^{-5}$ & 0.08 & 1.57 \\
\hline & (5.13) & & & $(0.29)$ & & $\left(1 \times 10^{-5}\right)$ & & \\
\hline \multirow[t]{2}{*}{$7 b$} & $20.84 * * *$ & & & & 0.01 & $4 \times 10^{-5}$ & 0.07 & 1.56 \\
\hline & $(5.08)$ & & & & $(0.03)$ & $\left(4 \times 10^{-5}\right)$ & & \\
\hline
\end{tabular}


$N=367$; Date range: 28 December 2011 - 23 May 2013. Models are estimated using generalized method of moments correcting for autocorrelation up to order 4. The last column, marked $D$ - $W$, reports Durbin-Watson statistics. Heteroscedasticity-consistent (Eicker-White) standard errors are in parentheses. $* * *=$ significant at $1 \%$; $* *=$ significant at $5 * ; *$ significant at $10 \%$.

Table 5. Regression Results, Japanese Yen

\begin{tabular}{|c|c|c|c|c|c|c|c|c|}
\hline & $\Delta X_{t}$ & $\Delta i_{t}^{L I B O R}$ & $\Delta i_{t}^{O C R}$ & $\Delta i_{t}^{L I B O R}$ & $\Delta i_{t}^{O C R}$ & TREND & $R^{2}$ & $D-W$ \\
\hline \multirow{2}{*}{$1 \mathrm{a}$} & $24.44 *$ & & & -0.31 & & & 0.05 & 2.10 \\
\hline & (12.90) & & & 0.94 & & & & \\
\hline \multirow{2}{*}{$1 b$} & $24.26 * *$ & & & & & & 0.05 & 2.10 \\
\hline & (12.70) & & & & $(0.59)$ & & & \\
\hline \multirow[t]{2}{*}{2} & & 0.30 & & & & & 0.00 & 2.05 \\
\hline & & (2.05) & & & & & & \\
\hline \multirow[t]{2}{*}{$3 a$} & & & & & & & 0.00 & 2.05 \\
\hline & & & $(0.24)$ & & & & & \\
\hline \multirow{2}{*}{$3 b$} & & & & -0.33 & & & 0.00 & 2.05 \\
\hline & & & & $(1.00)$ & & & & \\
\hline \multirow[t]{2}{*}{$4 a$} & & & & & -0.54 & & 0.00 & 2.05 \\
\hline & & & & & (0.59) & & & \\
\hline \multirow{2}{*}{$4 b$} & $24.15^{*}$ & -0.29 & & & & $1 \times 10^{-4}$ & 0.05 & 2.10 \\
\hline & (13.10) & (1.92) & & & & $\left(1 \times 10^{-4}\right)$ & & \\
\hline \multirow[t]{2}{*}{$5 a$} & $23.86^{*}$ & & 0.11 & & & $1 \times 10^{-4}$ & 0.05 & 2.10 \\
\hline & (13.00) & & $(0.28)$ & & & $\left(1 \times 10^{-4}\right)$ & & \\
\hline \multirow{2}{*}{$5 b$} & $23.99 *$ & & & -0.31 & & $1 \times 10^{-4}$ & 0.04 & 2.11 \\
\hline & (12.83) & & & $(0.92)$ & & $\left(1 \times 10^{-4}\right)$ & & \\
\hline \multirow[t]{2}{*}{$6 a$} & $23.98^{*}$ & & & & -0.33 & $1 \times 10^{-4}$ & 0.05 & 2.11 \\
\hline & (12.72) & & & & $(0.47)$ & $\left(1 \times 10^{-4}\right)$ & & \\
\hline \multirow[t]{2}{*}{$6 b$} & $24.44 *$ & & & -0.31 & & & 0.05 & 2.10 \\
\hline & (12.90) & & & 0.94 & & & & \\
\hline \multirow[t]{2}{*}{$7 \mathrm{a}$} & $24.26 * *$ & & & & -0.52 & & 0.05 & 2.10 \\
\hline & (12.70) & & & & (0.59) & & & \\
\hline \multirow[t]{2}{*}{$7 b$} & & 0.30 & & & & & 0.00 & 2.05 \\
\hline & & $(2.05)$ & & & & & & \\
\hline
\end{tabular}

$N=177$; Date range: 12 September 2012 - 23 May 2013. Models are estimated using generalized method of moments correcting for autocorrelation up to order 4. The last column, marked $D-W$, reports Durbin-Watson statistics. Heteroscedasticity-consistent (Eicker-White) standard errors are in parentheses. $* * *=$ significant at $1 \%$; $* *=$ significant at $5 * ;=$ significant at $10 \%$.

\section{Conclusion and Caveats}

This paper applies an FX microstructure model to the Bitcoin market. As with all applications of FX microstructure, our key variable is order flow, which acts as the conduit from often-heterogenous information to market behavior and discovery. Given the lack of meaningful Bitcoin-denominated fundamentals, the microstructure approach is the only 
way to avoid a pure time-series analysis of this market; accordingly, applying microstructure modeling techniques to the Bitcoin market represents a "back to basics" approach to analyze a relatively young asset that is in the early stages of development. As in prior studies employing microstructure methods on standard currency markets, we find that order flow accounts for almost all or all of the explanatory power for changes in the Bitcoin exchange rate. Our strongest result finds that order flow accounts over $40 \%$ of the daily change in the U.S. dollar/Bitcoin spot rate.

This work makes two contributions. First, our results suggest that although Bitcoin is very different than standard sovereign currencies, Bitcoin's information structure and the way information is transmitted to prices is similar to that found in regular FX markets. In other words, Bitcoin prices behave like those of any other currency in most respects. As continued growth in and acceptance of Bitcoin as a currency and an asset is likely dependent on brokerage other intermediation services becoming more readily available, this bodes well for the future of private digital currencies.

Second, they illustrate the general robustness, and hence relevance of the microstructure approach. As noted, Bitcoin spot rates are much more volatile than standard exchange rates, a by-product of Bitcoin's age and a series of idiosyncratic, market-disrupting shocks during our sample period. We would argue that being able to estimate a model that had any explanatory power for this unsettled market is meaningful per se. And to deliver results for Bitcoin that compare favorably with analogous research on traditional currencies is, in our opinion, consequential. Going forward, we expect that the microstructure approach will continue to be an area of active and influential scholarship for traditional currencies, non-monetary financial assets, and digital currencies like Bitcoin.

Finally, a few caveats about our findings are in order. We have noted that the Bitcoin market is in the early stages of development. As (or if) this market matures, it is entirely possible, nay probable that the behavior of the Bitcoin spot rate could change. With maturity brings decreased volatility, for example, and it is possible that Bitcoin-denominated assets could be introduced. Anecdotally, one could argue that the typical Bitcoin investor differs from the typical currency trader. The counterculture aspect of Bitcoin remains a selling point, and anecdotally, individual Bitcoin enthusiasts are still a big part of this market and individual trades tend to be small. We expect this to change going forward, as more professional investors are drawn into this market. We also note that Bitcoin brokers and dealers are becoming much more commonplace. In all, the structure of the Bitcoin market stands to change considerably going forward, and we stress that the conclusions presented here apply only to the early stages of Bitcoin's development. That said, we anticipate that the microstructure FX approach will remain the best option to study this market in the future.

\section{Acknowledgements}

We would like to thank Zachary Littrell for valuable research assistance; and Julie Routzahn and seminar participants at Université Saint-Louis, Brussels and Anhui Normal University, Wuhu, China for helpful comments and suggestions. All errors are ours.

\section{References}

Carlson, J. A., \& Lo, M. (2006). One Minute in the Life of the DM/US\$: Public News in an Electronic Market. Journal of International Money and Finance, 25(7), 1090-1102.

Cerrato, M., Sarantis, N., \& Saunders, A. (2011). An Investigation of Customer Order Flow in the Foreign Exchange Market. Journal of Banking \& Finance, 35(8), 1892-1906.

Chinn, M. D., \& Moore, M. J. (2011). Order Flow and the Monetary Model of the Exchange Rates: Evidence from a Novel Data Set. Journal of Money, Credit, and Banking, 48(8), 1599-1625.

Daníelsson, J., Luo, J., \& Payne, R. (2012). "Exchange Rate Determination and Inter-market Order Flow Effects." European Journal of Finance, 18(9-10), 823-840.

Ding, L., \& Ma, J. (2013). "Portfolio Reallocation and Exchange Rate Dynamics." Journal of Banking \& Finance 37, 3100-3124.

Evans, M. D. D. \& Lyons, R. K. (2002). “Order Flow and Exchange Rate Dynamics.” Journal of Political Economy 110(1) (February), 170-180.

Evans, M. D. D. (2010). “Order Flows and The Exchange Rate Disconnect Puzzle.” Journal of International Economics 80(1), 58-71.

Evans, M. D. D. (2011). Exchange Rate Dynamics. Princeton, NJ and Oxford: Princeton University Press.

Evans, M. D. D., \& Lyons, R. K. (2005). "Do Currency Markets Absorb News Quickly?” Journal of International Money and Finance, 24(2), 197-217.

Evans, M. D. D., \& Lyons, R. K. (2008). "How Is Macro News Transmitted to Exchange Rates?” Journal of Financial Economics, 88(1), 26-50. 
Frankel, J. A., \& Rose, A. K. (1995). "Empirical Research on Nominal Exchange Rates." Handbook of International Economics, 3, G. Grossman and K. Rogoff, eds. Amsterdam, NL: Elsevier.

Grinberg, R. (2011). "Bitcoin: An Innovative Alternative Digital Currency." Hastings Science and Technology Law Journal, 4(1), 159-208.

King, M. R., Osler, C. L., \& Rime, D. (2013). "The Market Microstructure Approach to Foreign Exchange: Looking Back and Looking Forward.” Journal of International Money and Finance, 38, 95-119.

Kitamura, Y. (2011). "The Impact of Order Flow on the Foreign Exchange Market: A Copula Approach.” Asia-Pacific Financial Markets, 18, 1-31.

Lovcha, Y., \& Perez-Laborda, A. (2010). "Is [the] Exchange Rate-Customer Order Flow Relationship Linear? Evidence From the Hungarian FX Market.” MNB Working Papers, \#2010/10.

Marsh, I. W., \& O'Rourke, C. (2005). "Customer Order Flow and Exchange Rate Movements: Is There Really Information Content?" Norges Bank Conference on Equity and Currency Microstructure, Oslo.

Meese, R. A., \& Rogoff, K. “Empirical Exchange Rate Models of the Seventies: Do They Fit Out of Sample?” Journal of International Economics, 14(1/2), 3-24.

Menkhoff, L., Sarno, L., Schmeling, A., \& Schrimph, A. (2012). The Cross-Section of Currency Order Flow Portfolios. Working paper.

Nakamoto, S. (2008). "Bitcoin: A Peer-to-Peer Electronic Cash System.” online manuscript. Satoshi Nakamoto Institute, http://www.nakamotoinstitute.org/bitcoin/.

Plassaras, N. A. (2013). "Regulating Digital Currencies: Bringing Bitcoin within the Reach of the IMF." Chicago Journal of Intemational Law, Summer 2013, 377-407.

Reitz, S., Schmidt, M. A., \& Taylor, M. P. (2007). "End-user Order Flow and Exchange Rate Dynamics." Deutsche Bundesbank Discussion Paper Series 1: Economic Studies \#05/2007.

Riasi, A. (2015). "Competitive Advantages of Shadow Banking Industry: An Analysis Using Porter Diamond Model." Business Management and Strategy 6(2), pp. 15-27. http://dx.doi.org/10.5296/bms.v6i2.8334

Sager, M., \& Taylor, M. P. (2008). "Commercially Available Order Flow Data and Exchange Rate Movements: Caveat Emptor." Journal of Money, Credit, and Banking, 40(4), 583-625.

Takemoto, Y., \& Knight, S. (2014). "Mt. Gox files for bankruptcy, blames hackers for losses." Reuters, February 28.

Velde, F. (2013). "Bitcoin: A Primer." Chicago Fed Letter \#317, December.

Yermack, D. (2013). “Is Bitcoin a Real Currency?” National Bureau of Economic Research (NBER) Working Papers $\# 19747$. 


\section{Notes}

Note 1. We adopt the convention of using "Bitcoin" in both the singular and irregular plural tense.

Note 2. A Bitcoin can be divided to the eighth decimal place, or one "Satoshi." Once divided, a Bitcoin cannot be reconstituted.

Note 3. Prior to 2016, additions to the blockchain occurred approximately every ten minues. 25 new Bitcoin are mined per new block added. In 2016 this amount will be halved to 12.5 Bitcoin per new block and halved again in 2020 and every four years thereafter for the next 100-130 years, until the total supply of Bitcoin reaches 21 million (Grinberg, 2011).

Note 4. Interest in Bitcoin went hand-in-hand with publicity generated by the now-defunct Silk Road, a notorious deep-web marketplace dealing in illicit drugs and other black market wares. At its height, Silk Road had an estimated sales volume of roughly U.S. $\$ 15$ million, all conducted in Bitcoin. The original Silk Road website was taken down by U.S. authorities in late 2013.

Note 5. Interestingly, the Chinese renmimbi and various developing-world currencies are accounting for an incresing share of Bitcoin trading volume since 2013.

Note 6. See, for example, the seminal work by Meese and Rogoff (1983) and Frenkel and Rose's (1995) summary.

Note 7. FX microstructure models are typically specified at weekly or daily frequencies, whereas most estimates of inflation and output-related fundamentals are released on a monthly basis in most countries. This makes interest rates the fundamental of choice by default. One noteworty exception is found in recent work by Evans (2010), who develops real-time, high-frequency estimates of GDP, the money supply, and inflation.

Note 8. The latter observation-relatively high kurtosis—is consistent with Menkhoff et al. (2012) who find similar results for customer order flow in standard currency trading.

\section{(cc) EY}

This work is licensed under a Creative Commons Attribution 3.0 License. 